\title{
Qualidade de vida e fatores associados em trabalhadores de uma Universidade do Sul de Santa Catarina
}

\section{Quality of life and factors associated of University employees from southern Santa Catarina State}

\author{
Fernanda de Oliveira Meller* (ㄱ) Antonio José Grande², Micaela Rabelo Quadra³, \\ Ramiro Doyenart ${ }^{4}$, Antônio Augusto Schäfer ${ }^{1}$ (D) \\ ${ }^{1}$ Programa de Pós-graduação em Saúde Coletiva, Universidade do Extremo Sul Catarinense (UNESC) - Criciúma (SC), Brasil. \\ ${ }^{2}$ Curso de Medicina, Universidade Estadual de Mato Grosso do Sul (UEMS) - Campo Grande (MS), Brasil. \\ ${ }^{3}$ Curso de Nutrição, Universidade do Extremo Sul Catarinense (UNESC) - Criciúma (SC), Brasil. \\ ${ }^{4}$ Curso de Educação Física, Universidade do Extremo Sul Catarinense (UNESC) - Criciúma (SC), Brasil.
}

Como citar: Meller FO, Grande AJ, Quadra MR, Doyenart R, Schäfer AA. Qualidade de vida e fatores associados em trabalhadores de uma Universidade do Sul de Santa Catarina. Cad Saúde Colet, 2020;28(1):87-97. https://doi.org/10.1590/1414-462X202028010327

\section{Resumo}

Introdução: A qualidade de vida de trabalhadores interfere no desenvolvimento e na produtividade do trabalho e pode ser influenciada por diversos fatores sociodemográficos e laborais. Objetivo: Avaliar os fatores associados à qualidade de vida de funcionários de uma Universidade. Método: Estudo transversal realizado com 214 trabalhadores de ambos os sexos pertencentes aos dezenove setores da Universidade. Para avaliar a qualidade de vida, utilizou-se o WHOQOL-Bref. A associação de cada domínio da qualidade de vida com as variáveis de exposição foi avaliada através do teste t para amostras independentes ou análise de variância, seguida do teste de Bonferroni. Resultados: As médias dos domínios de qualidade de vida foram: 74,64 $( \pm 13,52)$ para o físico, $71,12( \pm 12,85)$ para o psicológico, 76,94 $( \pm 13,98)$ para o de relações sociais e $61,94( \pm 16,30)$ para o ambiente. Os homens apresentaram maiores médias para os domínios físico, psicológico e de relações sociais. Trabalhadores com idade maior do que 38 anos apresentaram maiores médias no domínio psicológico. Já no domínio relações sociais, as maiores médias foram observadas entre os indivíduos de 18 a 27 anos. Naqueles indivíduos que dormiam menos de 8 horas por dia, as médias do domínio físico foram menores. Conclusão: Fazem-se necessárias ações de prevenção e promoção da qualidade de vida no trabalho, especialmente direcionadas aos funcionários que apresentaram as menores médias nos domínios de qualidade de vida.

Palavras-chave: qualidade de vida; fatores de risco; saúde do trabalhador; estudos transversais.

\begin{abstract}
Background: Life quality of workers influences on development and productivity of work and it can be influenced by several sociodemographic and labor factors. Objective: To evaluate the associated factors with quality of life of University workers from southern Santa Catarina State. Method: A cross-sectional study with 214 workers was carried out. WHOQOL-Bref was used to evaluate the quality of life. The quality of life domains were associated to exposure variables. The statistical analysis T-test for independent samples and analysis of variance, followed by the Bonferroni test were used. Results: The mean of quality of life domains were: $74.64( \pm 13.52)$ for the physical domain, $71.12( \pm 12.85)$ for the psychological, $76.94( \pm 13.98)$ for social relations domain and $61.94( \pm 16.30)$ for the environment domain. Males presented higher mean for the physical, psychological and social relations domains. Workers older than 38 years of age presented higher means in the psychological domain. In the social relations domain, the highest mean
\end{abstract}

Este é um artigo publicado em acesso aberto (Open Access) sob a licença Creative Commons Attribution, que permite uso, distribuição e reprodução em qualquer meio, sem restrições desde que o trabalho original seja corretamente citado.
Trabalho realizado na Universidade do Extremo Sul Catarinense (UNESC) - Criciúma (SC), Brasil.

Correspondência: Fernanda de Oliveira Meller. E-mail: fernandameller@unesc.net

Fonte de financiamento: nenhuma.

Conflito de interesses: nada a declarar.

Recebido em: Ago. 15, 2018. Aprovado em: Maio 25, 2019 
was observed among individuals aged 18 to 27 years. In those individuals who slept less than 8 hours a day, the mean of the physical domain were smaller. Conclusion: It is necessary the development of actions to prevent and promote life quality at work focusing on employees who had the lowest averages of the domains of that.

Keywords: life quality; risk factors; employees; cross-sectional study.

\section{INTRODUÇÃO}

De acordo com a Organização Mundial da Saúde (OMS), qualidade de vida conceitua-se como a percepção que cada indivíduo possui em relação a sua disposição na vida: sua cultura, objetivos, padrões, preocupações e expectativas'. Já o Ministério da Saúde a define como o estado de satisfação das necessidades básicas dos seres humanos, como a alimentação, a educação, a habitação e o acesso a água potável².

A qualidade de vida dos trabalhadores pode estar associada a diversos fatores ${ }^{3-5}$, sendo o principal deles o ambiente laboral ${ }^{5}$. De acordo com a literatura, condições físicas e instalações inadequadas e deficitárias, excesso de funções burocráticas, normas e procedimentos administrativos inadequados, remuneração insuficiente, longas jornadas de trabalho, falta de reconhecimento e desvalorização profissional podem tornar os funcionários menos motivados e, consequentemente, influenciar negativamente a qualidade de vida ${ }^{3,6}$.

Além disso, estudos têm evidenciado que variáveis sociodemográficas como sexo, idade e escolaridade interferem na qualidade de vida dos trabalhadores. Indivíduos do sexo feminino e com menor escolaridade tendem a apresentar menores médias para a maioria dos domínios de qualidade de vida, ou seja, físico, psicológico e de relações sociais, enquanto os adultos jovens apresentam maior média para o domínio de relações sociais ${ }^{5,7-12}$. Outro fator que tem papel importante na qualidade de vida de trabalhadores é o sono. De acordo com Marconato et al. (2015), existe uma associação direta entre horas de sono e domínio físico ${ }^{13}$.

Diante do exposto, torna-se fundamental estudar a qualidade de vida no ambiente laboral, uma vez que a avaliação da segurança física, mental, social, da saúde e do bem-estar permite identificar a satisfação pessoal do trabalhador, o que interfere na qualidade, no desenvolvimento e também na produtividade do trabalho ${ }^{14}$. Assim, o presente estudo objetivou avaliar a qualidade de vida e seus fatores associados em funcionários de uma Universidade do Sul de Santa Catarina.

\section{MÉTODO}

Estudo transversal realizado com trabalhadores de uma Universidade comunitária do Sul de Santa Catarina, Brasil, no período de dezembro de 2016 a janeiro de 2017.

A amostra foi calculada considerando o número total de trabalhadores da Universidade $(n=584)$, prevalência do desfecho de $50 \%$ e nível de confiança de $95 \%$, totalizando 232 pessoas. Após, acrescentou-se 15\% para perdas e recusas, finalizando a amostra em 267 funcionários.

Amostragem proporcional por setores de trabalho da instituição foi realizada e, posteriormente, dentro de cada setor (total de 19), sortearam-se, aleatoriamente, os trabalhadores a serem entrevistados.

Foi aplicado, a todos os funcionários que participaram do estudo, um questionário contendo informações demográficas, socioeconômicas, antropométricas e comportamentais. Para avaliar a qualidade de vida dos trabalhadores, foi utilizado oWHOQOL-Bref, versão abreviada do WHOQOL-100. Este instrumento avalia a qualidade de vida em quatro domínios, sendo eles: físico, psicológico, relações sociais e ambiente. No domínio físico estão englobadas questões referentes a atividades de vida diária, dependência de substâncias medicinais e ajuda médica, energia e fadiga, mobilidade, dor e desconforto, sono e repouso, e capacidade para o trabalho. O domínio psicológico inclui imagem corporal e aparência, sentimentos positivos e negativos, autoestima, espiritualidade/religião/crenças pessoais, pensamento, aprendizagem, memória e concentração. No domínio relações sociais estão incorporadas questões sobre relações pessoais, suporte social e atividade sexual. Por fim, o domínio ambiente inclui recursos financeiros, liberdade, segurança, cuidado social e de saúde (acesso e qualidade), ambiente domiciliar, 
oportunidades para adquirir novas informações e habilidades, participação e oportunidades de recreação/atividade de lazer, ambiente físico (poluição/barulho/tráfego/clima) e transporte. O instrumento é composto por 26 questões, e a média do escore de cada domínio varia de 0 a 100. Quanto maior o escore, melhor a qualidade de vidaa ${ }^{15,16}$.

Esses questionários foram aplicados por entrevistadores treinados. Após a coleta dos dados, foi realizada dupla digitação no programa Excel versão 2010.

Para avaliar o estado nutricional dos entrevistados, foi calculado o índice de massa corporal $(\mathrm{IMC})^{17}$ utilizando o peso e a altura autorreferidos. Os indivíduos com IMC $<25 \mathrm{~kg} / \mathrm{m}^{2}$ foram classificados como "sem excesso de peso", e aqueles com IMC $\geq 25 \mathrm{~kg} / \mathrm{m}^{2}$ como "com excesso de peso". A duração o sono foi avaliada em horas por dia da semana, sendo dicotomizada em " $<8$ horas/dia" e " $\geq 8$ horas/dia". As variáveis de exposição demográficas e socioeconômicas incluídas na análise foram sexo (masculino/feminino), idade (18-27, 28-37, 38-47, 48-60 anos), estado civil (solteiro(a), casado(a), separado(a)/divorciado(a), viúvo(a)) e escolaridade (0-4, 5-8, 9-11, 12 anos ou mais).

Foram realizadas análises descritivas das variáveis estudadas apresentando frequências absoluta e relativa das variáveis de exposição, além de seus respectivos IC95\%. Foram também apresentadas as médias e os desvios-padrão dos quatro domínios da qualidade de vida.

Além disso, foi avaliada a associação de cada domínio com as variáveis de exposição estudadas, através do teste t para amostras independentes (variável de exposição dicotômica) ou análise de variância (variável de exposição politômica), seguida do teste de Bonferroni para múltiplas comparações. A significância estatística utilizada foi $5 \%$.

Para a análise dos dados foi utilizado o programa estatístico Stata versão 12.1.

O presente estudo foi aprovado pelo Comitê de Ética em Pesquisa da Universidade do Extremo Sul Catarinense sob protocolo no 59682816.3.0000.0119. Todos os participantes que aceitaram participar do estudo assinaram um Termo de Consentimento Livre e Esclarecido.

\section{RESULTADOS}

Dos 267 indivíduos elegíveis para o estudo, considerando o cálculo amostral mais 15\%, 53 não participaram por não aceitarem ou não terem sido encontrados, sendo desenvolvido, então, com 214 participantes. A Figura 1 apresenta o fluxograma dos participantes do estudo.

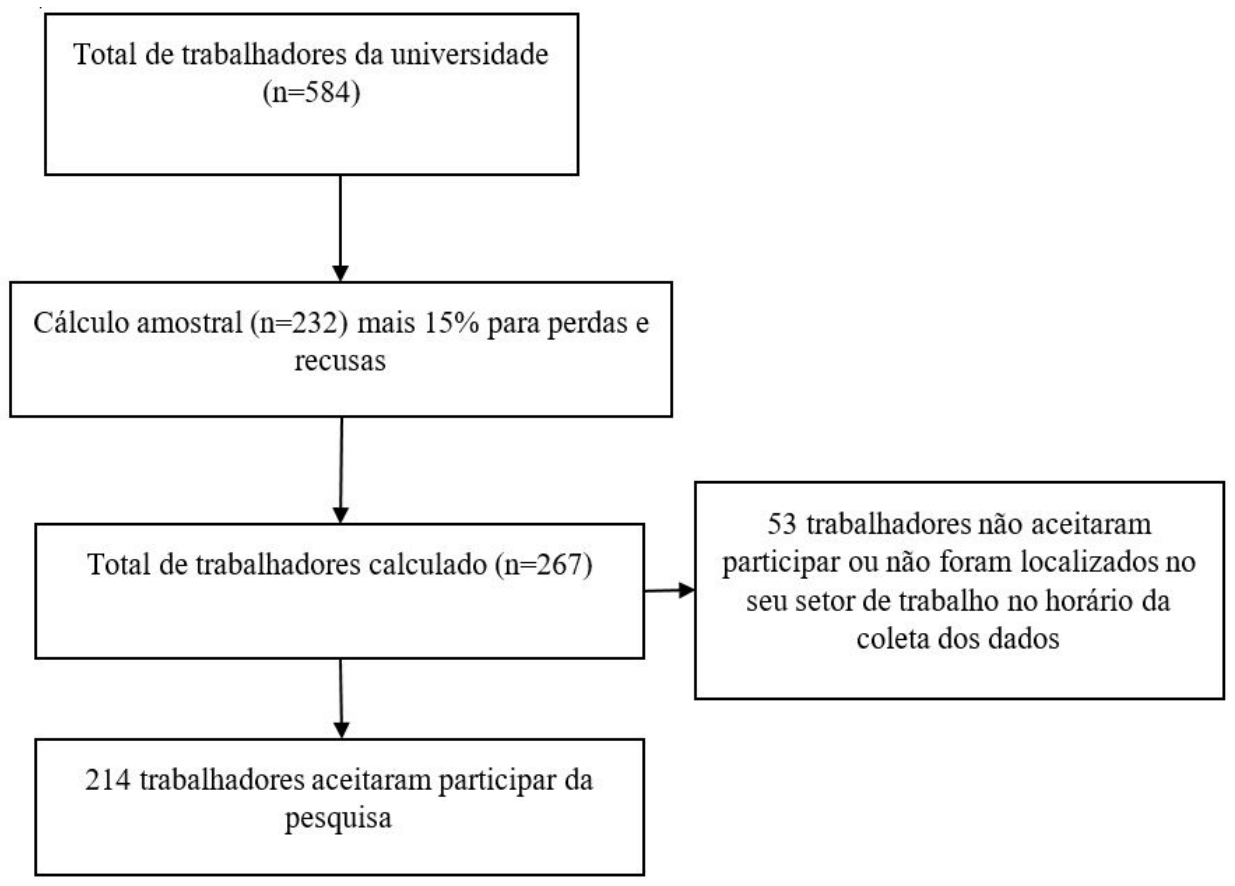

Figura 1. Fluxograma dos participantes do estudo 
Na Tabela 1 estão apresentadas as características dos trabalhadores estudados. Pode-se observar que a grande maioria deles era do sexo feminino $(65,3 \%$; IC $95 \% 58,8-71,7)$ e tinha 18 a 37 anos de idade $(66,3 \%$, IC95\% 60,0-72,7). Quase metade dos entrevistados era solteiro $(44,4 \%$, IC95\% 37,7-51,1) e tinha, no mínimo, doze anos de estudo $(47,5 \%$, IC95\% 40,8-54,4). Mais da metade da amostra tinha excesso de peso $(54,9 \%$, IC\% 48,0-61,8) e dormia menos de 8 horas por dia $(67,1 \%$, IC95\% 60,8-73,5).

Tabela 1. Características demográficas, socioeconômica, antropométrica e comportamental dos trabalhadores de uma Universidade do Sul de Santa Catarina, 2016/2017. (n=214)

\begin{tabular}{|c|c|c|}
\hline Variáveis & $\mathbf{n}$ & \% (IC95\%) \\
\hline \multicolumn{3}{|l|}{ Sexo } \\
\hline Masculino & 74 & $34,7(28,3-41,2)$ \\
\hline Feminino & 139 & $65,3(58,8-71,7)$ \\
\hline \multicolumn{3}{|l|}{ Idade (em anos) } \\
\hline $18-27$ & 70 & $32,7(26,4-39,0)$ \\
\hline $28-38$ & 73 & $34,1(27,7-40,5)$ \\
\hline$>38$ & 71 & $33,2(26,8-39,5)$ \\
\hline \multicolumn{3}{|l|}{ Estado civil } \\
\hline Solteiro & 95 & $44,4(37,7-51,1)$ \\
\hline Casado & 99 & $46,2(39,5-53,0)$ \\
\hline Separado/divorciado/viúvo & 20 & $9,4(5,4-13,3)$ \\
\hline \multicolumn{3}{|l|}{ Escolaridade (em anos completos) } \\
\hline $0-8$ & 24 & $11,5(7,1-15,8)$ \\
\hline $9-11$ & 86 & $41,0(34,2-47,7)$ \\
\hline 12 ou mais & 100 & $47,5(40,8-54,4)$ \\
\hline \multicolumn{3}{|l|}{ Estado nutricional } \\
\hline Sem excesso de peso & 92 & $45,1(38,2-52,0)$ \\
\hline Com excesso de peso & 112 & $54,9(48,0-61,8)$ \\
\hline \multicolumn{3}{|l|}{ Duração do sono (horas/dia) } \\
\hline$<8$ & 143 & $67,1(60,8-73,5)$ \\
\hline 8 ou mais & 70 & $32,9(26,5-39,2)$ \\
\hline
\end{tabular}

Percentual máximo de observações desconhecidas para a variável escolaridade: $4,7 \%(n=10)$

A Tabela 2 mostra as médias dos quatro domínios de qualidade de vida dos trabalhadores, sendo elas: 74,64 ( $\pm 13,52)$ para o domínio físico, $71,12( \pm 12,85)$ para o psicológico, $76,94( \pm 13,98)$ para o de relações sociais e $61,94( \pm 16,30)$ para o domínio ambiente. 
Tabela 2. Média e desvio-padrão dos domínios de qualidade de vida dos trabalhadores de uma Universidade do Sul de Santa Catarina, 2016/2017. (n=214)

\begin{tabular}{lccc}
\multicolumn{1}{c}{ Domínios } & $\mathbf{n}$ & Média & Desvio-padrão \\
\hline Físico & 213 & 74,64 & 13,52 \\
\hline Psicológico & 210 & 71,12 & 12,85 \\
\hline Relações sociais & 211 & 76,94 & 13,98 \\
\hline Ambiente & 213 & 61,94 & 16,30 \\
\hline
\end{tabular}

Percentual máximo de observações desconhecidas para o domínio aspecto psicológico: 1,9\% (n=4)

A associação entre os domínios e as variáveis de exposição estudadas está apresentada na Tabela 3. Pode-se observar que o sexo masculino apresentou maiores médias para os domínios físico $(p=0,001)$, psicológico $(p<0,001)$ e de relações sociais $(p<0,001)$, quando comparado ao sexo feminino. A variável idade também se mostrou associada aos domínios psicológico e relações sociais. Aqueles trabalhadores com idade maior do que 38 anos apresentaram maiores médias no domínio psicológico do que aqueles com idade entre 28 e 38 anos ( $p=0,029$ ). No domínio relações sociais, as maiores médias foram observadas entre os indivíduos de 18 a 27 anos de idade quando comparados àqueles com 28 a 38 anos $(p=0,035)$. Além disso, as médias do domínio físico foram menores nos indivíduos que dormiam menos de 8 horas por dia $(p=0,036)$. As demais variáveis não estiveram associadas com os domínios da qualidade de vida.

Tabela 3. Médias e desvio-padrão (DP) dos domínios da qualidade de vida conforme variáveis de exposição estudadas dos trabalhadores de uma Universidade do Sul de Santa Catarina, 2016/2017. (n=214)

\begin{tabular}{|c|c|c|c|c|c|c|c|c|}
\hline \multirow[b]{2}{*}{ Variáveis } & \multicolumn{2}{|c|}{ Físico } & \multicolumn{2}{|c|}{ Psicológico } & \multicolumn{2}{|c|}{ Relações sociais } & \multicolumn{2}{|c|}{ Ambiente } \\
\hline & $\begin{array}{l}\text { Média } \\
\text { (DP) }\end{array}$ & $\mathbf{p}$ & $\begin{array}{l}\text { Média } \\
\text { (DP) }\end{array}$ & $\mathbf{p}$ & $\begin{array}{l}\text { Média } \\
\text { (DP) }\end{array}$ & $\mathbf{p}$ & $\begin{array}{l}\text { Média } \\
\text { (DP) }\end{array}$ & $\mathbf{p}$ \\
\hline Sexo* & & 0,001 & & $<0,001$ & & $<0,001$ & & 0,363 \\
\hline Masculino & $\begin{array}{c}79,05 \\
(12,59)\end{array}$ & & $\begin{array}{l}75,58 \\
(9,88)\end{array}$ & & $\begin{array}{c}81,78 \\
(13,97)\end{array}$ & & $\begin{array}{c}63,24 \\
(17,61)\end{array}$ & \\
\hline Feminino & $\begin{array}{c}72,32 \\
(13,50)\end{array}$ & & $\begin{array}{c}68,66 \\
(13,57)\end{array}$ & & $\begin{array}{c}74,32 \\
(13,37)\end{array}$ & & $\begin{array}{c}61,10 \\
(15,54)\end{array}$ & \\
\hline Idade (em anos) ${ }^{* *}$ & & 0,114 & & 0,029 & & 0,035 & & 0,063 \\
\hline $18-27$ & $\begin{array}{c}76,38 \\
(12,62)\end{array}$ & & $\begin{array}{c}71,54 \\
(12,73)\end{array}$ & & $\begin{array}{c}79,21 \\
(14,61)^{* * *}\end{array}$ & & $\begin{array}{c}63,70 \\
(16,31)\end{array}$ & \\
\hline $28-38$ & $\begin{array}{l}72,00 \\
(13,61)\end{array}$ & & $\begin{array}{c}68,13 \\
(11,61)^{* * *}\end{array}$ & & $\begin{array}{c}73,51 \\
(12,99)^{* * *}\end{array}$ & & $\begin{array}{c}58,32 \\
(16,42)\end{array}$ & \\
\hline$>38$ & $\begin{array}{c}75,68 \\
(14,05)\end{array}$ & & $\begin{array}{c}73,80 \\
(13,70)^{* * *}\end{array}$ & & $\begin{array}{c}78,16 \\
(13,82)\end{array}$ & & $\begin{array}{c}63,96 \\
(15,74)\end{array}$ & \\
\hline Estado civil ${ }^{* *}$ & & 0,435 & & 0,516 & & 0,165 & & 0,778 \\
\hline Solteiro & $\begin{array}{c}75,98 \\
(13,51)\end{array}$ & & $\begin{array}{l}72,27 \\
(12,79)\end{array}$ & & $\begin{array}{c}78,65 \\
(14,32)\end{array}$ & & $\begin{array}{c}62,80 \\
(16,64)\end{array}$ & \\
\hline Casado & $\begin{array}{c}73,58 \\
(13,68)\end{array}$ & & $\begin{array}{c}70,16 \\
(13,51)\end{array}$ & & $\begin{array}{c}76,22 \\
(14,00)\end{array}$ & & $\begin{array}{c}61,13 \\
(16,46)\end{array}$ & \\
\hline $\begin{array}{l}\text { Separado/divorciado/ } \\
\text { viúvo }\end{array}$ & $\begin{array}{c}73,50 \\
(12,89)\end{array}$ & & $\begin{array}{l}70,45 \\
(9,57)\end{array}$ & & $\begin{array}{l}72,55 \\
(11,39)\end{array}$ & & $\begin{array}{c}61,80 \\
(14,27)\end{array}$ & \\
\hline
\end{tabular}

*Utilizado teste t para amostras independentes; ** Utilizada análise de variância; *** Teste Bonferroni para múltiplas comparações $(p<0,05)$ 
Tabela 3. Continuação...

\begin{tabular}{|c|c|c|c|c|c|c|c|c|}
\hline \multirow[b]{2}{*}{ Variáveis } & \multicolumn{2}{|c|}{ Físico } & \multicolumn{2}{|c|}{ Psicológico } & \multicolumn{2}{|c|}{ Relações sociais } & \multicolumn{2}{|c|}{ Ambiente } \\
\hline & $\begin{array}{l}\text { Média } \\
\text { (DP) }\end{array}$ & p & $\begin{array}{l}\text { Média } \\
\text { (DP) }\end{array}$ & $\mathbf{p}$ & $\begin{array}{l}\text { Média } \\
\text { (DP) }\end{array}$ & p & $\begin{array}{l}\text { Média } \\
\text { (DP) }\end{array}$ & $\mathbf{p}$ \\
\hline $\begin{array}{l}\text { Escolaridade (em anos } \\
\text { completos) }\end{array}$ & & 0,511 & & 0,902 & & 0,916 & & 0,081 \\
\hline $0-8$ & $\begin{array}{l}73,48 \\
(14,72)\end{array}$ & & $\begin{array}{c}71,52 \\
(14,20)\end{array}$ & & $\begin{array}{c}77,33 \\
(11,90)\end{array}$ & & $\begin{array}{c}57,35 \\
(18,86)\end{array}$ & \\
\hline $9-11$ & $\begin{array}{c}73,61 \\
(15,05)\end{array}$ & & $\begin{array}{c}71,69 \\
(14,62)\end{array}$ & & $\begin{array}{c}77,53 \\
(15,23)\end{array}$ & & $\begin{array}{c}60,42 \\
(17,45)\end{array}$ & \\
\hline 12 ou mais & $\begin{array}{l}75,78 \\
(12,04)\end{array}$ & & $\begin{array}{c}70,84 \\
(10,99)\end{array}$ & & $\begin{array}{c}76,67 \\
(13,53)\end{array}$ & & $\begin{array}{c}64,52 \\
(14,53)\end{array}$ & \\
\hline Estado nutricional* & & 0,675 & & 0,119 & & 0,882 & & 0,972 \\
\hline Sem excesso de peso & $\begin{array}{l}75,14 \\
(12,71)\end{array}$ & & $\begin{array}{c}69,48 \\
(13,51)\end{array}$ & & $\begin{array}{c}76,81 \\
(15,42)\end{array}$ & & $\begin{array}{c}62,25 \\
(16,68)\end{array}$ & \\
\hline Com excesso de peso & $\begin{array}{c}74,36 \\
(13,73)\end{array}$ & & $\begin{array}{c}72,34 \\
(12,28)\end{array}$ & & $\begin{array}{c}77,11 \\
(12,83)\end{array}$ & & $\begin{array}{c}62,17 \\
(15,83)\end{array}$ & \\
\hline $\begin{array}{l}\text { Duração do sono } \\
\text { (horas/dia)* }\end{array}$ & & 0,036 & & 0,815 & & 0,672 & & 0,868 \\
\hline$<8$ & $\begin{array}{c}73,35 \\
(14,25)\end{array}$ & & $\begin{array}{c}71,27 \\
(13,65)\end{array}$ & & $\begin{array}{c}77,24 \\
(14,68)\end{array}$ & & $\begin{array}{l}61,80 \\
(16,56)\end{array}$ & \\
\hline 8 ou mais & $\begin{array}{c}77,49 \\
(11,51)\end{array}$ & & $\begin{array}{c}70,83 \\
(11,13)\end{array}$ & & $\begin{array}{l}76,37 \\
(12,66)\end{array}$ & & $\begin{array}{c}62,20 \\
(15,98)\end{array}$ & \\
\hline
\end{tabular}

*Utilizado teste t para amostras independentes; ** Utilizada análise de variância; *** Teste Bonferroni para múltiplas comparações $(p<0,05)$

\section{DISCUSSÃO}

No presente estudo, foi possível observar que, entre os quatro domínios de qualidade de vida estudados, o que apresentou a maior média entre os funcionários foi de relações sociais, seguido dos domínios físico, psicológico e ambiente. Resultados similares foram encontrados por outros autores estudando trabalhadores de diferentes classes laborais $7,8,10,13,18$, o que indica uma tendência que se aplica a outras categorias profissionais ${ }^{10}$.

Assim como no presente estudo, o questionário utilizado por esses autores para avaliar a qualidade de vida dos trabalhadores foi o WHOQOL Bref, permitindo a comparabilidade dos achados com os do presente estudo. É importante destacar que tal questionário, desenvolvido pelo grupo World Health Organization Quality of Life, visa avaliar a qualidade de vida das populações, considerando aspectos como a percepção que as pessoas têm de si mesmas e de seu entorno, bem como a avaliação que cada pessoa faz de objetivos, expectativas, padrões e preocupações pessoais, dentro de um contexto cultural, social e ambiental ${ }^{19}$. Este instrumento permite a comparação entre estudos de diversos países e culturas, já que se encontra disponível em diferentes idiomas ${ }^{1}$.

O domínio de relações sociais atinge questões como relacionamentos, suporte e apoio'. O fato de este domínio ter apresentado a maior média nos estudos realizados com trabalhadores pode estar relacionado ao convívio saudável com os colegas de trabalho, o que acaba contribuindo para um suporte social satisfatório entre os trabalhadores ${ }^{20}$. No local onde foi desenvolvido o presente estudo, observa-se o suporte social fornecido também pelos gestores da Universidade, o que favorece relações saudáveis com os funcionários. Além disso, as diversas atividades integrativas frequentemente realizadas neste ambiente laboral podem ter contribuído para os resultados encontrados no estudo, uma vez que integra os funcionários de diferentes setores da Universidade. 
Uma possível explicação para o domínio ambiente ter apresentado a menor média seria que nesse domínio estão incluídas condições como lazer e educação ${ }^{10}$, além de acesso à saúde, segurança e infraestrutura do país ${ }^{13}$, que são dependentes de fatores sociais e também financeiros. De acordo com alguns autores, indivíduos com maior renda e maior escolaridade apresentam melhor qualidade de vida, principalmente no domínio ambiente ${ }^{10,18}$. Além disso, características do local de trabalho, como baixa iluminação, ruídos, falta de ventilação, elevadas horas de trabalho e mudanças climáticas, também influenciam negativamente o domínio ambiente ${ }^{21-23}$. Teles et al. ${ }^{24}$ trazem, ainda, que o comprometimento excessivo com o trabalho associado com a baixa realização e insatisfação laboral está relacionado com as menores médias no domínio ambiente, já que são capazes de afetar a percepção de um indivíduo sobre o ambiente em que vive ${ }^{24}$.

Em relação à associação entre os domínios da qualidade de vida e as variáveis de exposição estudadas, o presente estudo encontrou maiores médias nos domínios físico, psicológico e de relações sociais entre o sexo masculino. Não foi observada relação entre sexo e o domínio ambiente. Outros estudos encontraram resultados semelhantes ${ }^{6,8,10,25}$, evidenciando que os homens apresentaram médias mais elevadas para a maioria dos domínios de qualidade de vida.

De acordo com Araújo et al. ${ }^{26}$, além do trabalho profissional, as mulheres, muitas vezes, precisam se dedicar também às tarefas domésticas, matrimoniais e maternas ${ }^{26}$, o que pode prejudicar a construção de relações sociais dentro e fora do ambiente de trabalho ${ }^{8}$. Além disso, por questões físicas, elas estão mais expostas a sintomas osteomusculares e ao cansaço, especulando-se, assim, o porquê das menores médias nos domínios físico e de relações sociais entre o sexo feminino ${ }^{27}$. Além disso, os homens, geralmente, ocupam posições mais altas quando se refere ao ambiente profissional e, por consequência, recebem salários mais altos quando comparados com as mulheres ${ }^{26}$, o que pode explicar as maiores médias encontradas no domínio psicológico.

Corroborando os achados do presente estudo, alguns autores também evidenciaram que o sexo não influencia o domínio ambiente ${ }^{21-23,28}$. Kara ${ }^{28}$ demonstrou que homens e mulheres se preocupam igualmente com as necessidades econômicas, familiares, sociais e de conhecimento 28 .

Outro achado do presente estudo foi a associação entre idade e os domínios da qualidade de vida. Os trabalhadores com mais de 38 anos de idade, no domínio psicológico, apresentaram as maiores médias quando comparados aos trabalhadores de 28 a 38 anos. Já no domínio de relações sociais, observou-se a maior média nos trabalhadores entre 18 e 27 anos de idade em comparação àqueles com idade entre 28 e 38 anos. Tais achados corroboram aqueles encontrados por outros autores ${ }^{8,11}$. Os demais domínios, no presente estudo, não apresentaram associação com a variável idade.

Possível explicação para os resultados encontrados é que pessoas com mais idade demonstram ter maior satisfação com a vida quando comparadas àquelas mais jovens ${ }^{29}$. Segundo Steptoe et al. ${ }^{30}$, com o envelhecimento as pessoas se tornam mais sábias e seletivas, limitando suas atividades, eventos e experiências para aquilo que lhes é mais satisfatório emocionalmente, mantendo ou até mesmo melhorando o seu bem-estar psicológico ${ }^{30}$. Pesquisa sugere que indivíduos que ainda não são idosos apresentam mais sintomas de depressão e ansiedade ${ }^{31}$, enquanto os idosos apresentam uma melhora dos fatores relacionados à saúde mental ${ }^{31}$.

Por outro lado, Valinote et al. ${ }^{7}$ sugerem que pessoas acima dos 40 anos podem possuir maior média no domínio de relações sociais por serem mais maduras e manterem relacionamentos sólidos ${ }^{7}$. No entanto, segundo Souza e Hutz ${ }^{32}$, as pessoas procuram relacionamentos de acordo com o momento em que se encontram durante a vida e com as necessidades que possuem ${ }^{32}$. Estudo de revisão afirma que o tipo de relacionamento de amizade que o indivíduo possui, por exemplo, na adolescência, não será o mesmo mantido ao longo da vida adulta ${ }^{33}$. Durante o início da vida adulta, as pessoas se encontram no ápice dos relacionamentos sociais, pois ainda não assumiram papéis mais sérios que integram o seu ciclo de vida ${ }^{33}$, como o casamento e a criação de filhos. Estes papéis são costumeiramente mais desenvolvidos próximos à faixa 
etária dos 30 anos, o que condiz com a redução do número de relacionamentos sociais a partir desta idade ${ }^{33}$.

Muitas vezes, o aumento da idade e, consequentemente, o envelhecimento são acompanhados por problemas de saúde que podem afetar a qualidade de vida ${ }^{34}$. Por outro lado, segundo Almeida et al. ${ }^{29}$, a presença de dor e sua intensidade, assim como a ocorrência de acidentes de trabalho, não estão associadas à idade dos trabalhadores ${ }^{29}$. Este fato corrobora o presente estudo, que não encontrou associação entre idade e o domínio físico. Corroborando os achados do presente estudo, Nunes e Mascarenhas ${ }^{25}$ não encontraram associação entre a situação conjugal e os quatro domínios de qualidade de vida ${ }^{25}$. Já outro estudo observou influência do estado civil nos domínios de relações sociais e ambiente ${ }^{11}$. Segundo esta pesquisa, os indivíduos que eram casados ou moravam com o companheiro apresentaram a maior média no domínio de relações sociais, enquanto os indivíduos viúvos tiveram a maior média para o domínio ambiente ${ }^{11}$.

Alguns autores sugerem que ter um relacionamento pode, muitas vezes, significar plenitude pessoal e econômica. Além disso, um relacionamento saudável pode gerar bons sentimentos, autoestima, compartilhamento de situações cotidianas e melhores relações sociais ${ }^{35}$. Entretanto, o estado conjugal não parece ser capaz de influenciar grandemente os domínios da qualidade de vida, mesmo que ele possa sugerir companheirismo ou isolamento para as pessoas ${ }^{36}$.

A variável escolaridade também não esteve associada aos domínios de qualidade de vida dos funcionários do presente estudo, o que corrobora os achados de outros estudos ${ }^{7,25,37}$. Em contrapartida, no estudo de Mascarenhas et al. ${ }^{9}$, desenvolvido com profissionais de saúde, foi encontrada relação entre escolaridade e os domínios psicológico e relações sociais, apresentando menores médias os indivíduos que possuíam apenas o ensino fundamental ${ }^{9}$. Outro trabalho, realizado com enfermeiros, identificou que os indivíduos que tinham ensino superior apresentaram maiores médias no domínio ambiental ${ }^{12}$.

De acordo com Palhares et al. ${ }^{12}$, a menor escolaridade dos trabalhadores pode levar a um maior estresse no ambiente laboral ${ }^{12}$. Já segundo Valinote et al. ${ }^{7}$, quanto mais escolarizado é o indivíduo, maior o seu nível de estresse ${ }^{7}$, ou seja, mais crítico ele se torna, e os aspectos bons e ruins da sua vida se tornam mais nítidos ${ }^{36}$.

De acordo com Jahnke et al. ${ }^{38}$, o excesso de peso representa um risco para a saúde e para a ocorrência de acidentes relacionados ao ambiente laboral ${ }^{38}$, o que poderia afetar os domínios de qualidade de vida, uma vez que estão associados a questões como desconforto, dor, mobilidade e auxílio médico ${ }^{15}$. No entanto, é importante destacar que, no estudo de Marconato e Monteiro ${ }^{13}$, os indivíduos adultos com excesso de peso consideraram que seu estado nutricional não afetava a sua saúde, e relataram possuir uma saúde igual ou até mesmo melhor do que a das outras pessoas com a mesma faixa etária, porém sem excesso de peso ${ }^{13}$. Outros pesquisadores avaliaram a associação entre o percentual de gordura corporal e os quatro domínios de qualidade de vida, e constataram que a gordura corporal não influencia na qualidade de vida ${ }^{39}$. Tais achados corroboram os do presente estudo, que também não encontrou relação entre estado nutricional e qualidade de vida dos funcionários.

Outro achado do presente estudo é que os trabalhadores que dormiam menos de 8 horas por dia apresentaram menor média no domínio físico quando comparados àqueles que dormiam, pelo menos, 8 horas por dia. Os demais domínios não estiveram associados com a duração do sono. Similarmente, o estudo de Marconato e Monteiro ${ }^{13}$ encontrou correlação positiva entre horas de sono e domínio físico ${ }^{13}$. Os distúrbios no sono são capazes de afetar diretamente a qualidade de vida do indivíduo, podendo causar problemas de saúde, afetar o desempenho no ambiente de trabalho, o desenvolvimento de relacionamentos e a convivência ${ }^{40}$, além de dificultar a execução de tarefas diárias ${ }^{41}$. Segundo Pereira et al. ${ }^{41}$, pessoas que possuem qualidade de sono ruim podem apresentar, também, dor e desconforto ${ }^{41}$. As consequências da má qualidade do sono podem ser de curto, médio e longo prazo ${ }^{38}$. As primeiras consequências estão ligadas à parte biológica do organismo dos seres humanos e seu efeito é imediato, causando cansaço, mudanças no humor, fadiga, dificuldade de memória e atenção, ou seja, questões relacionadas ao domínio físico ${ }^{38}$. Logo após surgem as complicações 
funcionais em médio prazo, relacionadas ao desenvolvimento de atividades cotidianas, como o trabalho e relacionamentos ${ }^{38}$. Em longo prazo, as complicações afetam gravemente a qualidade de vida diária, podendo acarretar situações como problemas de saúde e perda de emprego ${ }^{38}$.

Estudos afirmam que pessoas com menor duração do sono possuem um risco aumentado de desenvolver doenças ${ }^{42}$, como a obesidade, uma vez que podem ter seu sistema endócrino alterado, passando a indicar de maneira aumentada a fome e o apetite ${ }^{43}$. Além disso, a privação constante do sono desregula o metabolismo dos carboidratos, podendo levar ao risco de desenvolver diabetes mellitus tipo $2^{44}$.

Diante dos resultados encontrados, pode-se concluir que os funcionários apresentaram menor média no domínio ambiente. Além disso, médias mais baixas para os domínios físico, psicológico e de relações sociais foram encontradas entre as mulheres. Os trabalhadores com idade entre 28 e 38 anos tiveram média inferior nos domínios psicológico e de relações sociais, e aqueles com duração do sono inferior a 8 horas por dia apresentaram menor média no domínio físico.

Considerando-se a importância da qualidade de vida na saúde dos indivíduos e sua influência no desenvolvimento e na produtividade no ambiente de trabalho, faz-se necessário o desenvolvimento de ações de prevenção e promoção da qualidade de vida no trabalho direcionadas aos funcionários que apresentaram as menores médias nos domínios de qualidade de vida.

\section{REFERÊNCIAS}

1. World Health Organization. WHOQOL: Measuring Quality of Life [Internet]. Geneva: WHO; 1997. [citado em 2018 junho 8]. Disponível em: http://www.who.int/iris/handle/10665/63482

2. Brasil. Ministério da Saúde. Glossário temático: promoção da saúde [Internet]. Brasília: Ministério da Saúde; 2013 [citado em 2018 junho 8]. Disponível em: http://bvsms.saude.gov.br/bvs/publicacoes/ glossario_tematico_promocao_saude.pdf

3. Anjara SG, Nellums LB, Bonetto C, Van Bortel T. Stress, health and quality of life of female migrant domestic workers in Singapore: a cross-sectional study. BMC Womens Health. 2017;17(1):1-13. http://dx.doi. org/10.1186/s12905-017-0442-7. PMid:29017558.

4. Vidotti HGM, Coelho VHM, Bertoncello D, Walsh IAP. Qualidade de vida e capacidade para o trabalho de bombeiros. Fisioter Pesqui. 2015;22(3):231-8.

5. Nahas MV. Atividade Física, Saúde e qualidade de vida: conceitos e sugestões para um estilo de vida ativo. Londrina: Midiograf; 2013.

6. Gomes KK, Sanchez HM, Sanchez EGM, Júnior ALS, Filho WMA, Silva LA, et al. Quality of life and quality of working life of health science professors at a higher education institution. Rev Bras Med Trab. 2017;15(1):1828. http://dx.doi.org/10.5327/Z1679443520177027.

7. Valinote HC, Pacheco LF, Viana FP, Formiga CKMR. Análise da qualidade de vida, capacidade para o trabalho e nível de estresse em trabalhadores da construção civil. RBCIAMB. 2014;32:115-26.

8. Pereira ÉF, Teixeira CS, Andrade RD, Lopes ADS. O trabalho docente e a qualidade de vida dos professores na educação básica. Rev Salud Publica. 2014;16(2):221-31. http://dx.doi.org/10.15446/rsap.v16n2.36484. PMid:25383496.

9. Mascarenhas $\mathrm{CHM}$, Prado FO, Fernandes $\mathrm{MH}$. Fatores associados à qualidade de vida de Agente Comunitários de Saúde. Cien Saude Colet. 2013;18(5):1375-86. http://dx.doi.org/10.1590/S141381232013000500023 . PMid:23670466.

10. Tabeleão VP, Tomasi E, Neves SF. Qualidade de vida e esgotamento profissional entre docentes da rede pública de ensino médio e fundamental no Sul do Brasil. Cad Saude Publica. 2011;27(12):2401-8. http:// dx.doi.org/10.1590/S0102-311X2011001200011. PMid:22218582.

11. Branco JC, Giusti PH, Almeida AR, Nichorn LF. Quality of life of employees of university hospital in Southern Brazil. J Health Sci Inst. 2010;28(2):199-203.

12. Palhares VC, Corrente JE, Matsubara BB. Association between sleep quality and quality of life in nursing professionals working rotating shifts. Rev Saude Publica. 2014;48(4):594-601. http://dx.doi.org/10.1590/ S0034-8910.2014048004939. PMid:25210818.

13. Marconato RS, Monteiro MI. Dor, percepção de saúde e sono: impacto na qualidade de vida de bombeiro/ profissionais do resgate. Rev Latinoam Enferm. 2015;23(6):991-99. 
14. Aquino AS, Fernandes ACP. Qualidade de vida no trabalho. J Health Sci Inst. 2013;31(1):53-8.

15. World Health Organization. WHOQOL-BREF Introduction, administration, scoring and generic version of the assessment [Internet]. Geneva: WHO; 1996 [citado em 2018 jun 8]. Disponível em: http://www.who. int/iris/handle/10665/63529.

16. Fleck MPA, Fachel O, Louzada S, Xavier M, Chachamovich E, Vieira G, et al. Desenvolvimento da versão em português do instrumento de avaliação de qualidade de vida da organização mundial da saúde (WHOQOL-100). Rev Bras Psiquiatr. 1999;21:19-28. http://dx.doi.org/10.1590/S1516-44461999000100006.

17. World Health Organization. Physical status: the use and interpretation of anthropometry. Geneva: WHO; 1995. (Report of a WHO Expert Committee. WHO Technical Report Series; 854).

18. Sorić M, Golubić R, Milosević M, Juras K, Mustajbegović J. Shift Work, quality of life and work ability among croatian hospital nurses. Coll Antropol. 2013;37(2):379-84. PMid:23940978.

19. World Health Organization. Quality of life assessment: international perspectives. Geneva: WHO; 1994.

20. Barros PCR, Mendes AMB. Sofrimento psíquico no trabalho e estratégias defensivas dos operários terceirizados da construção civil. Psico-USF. 2003;8(1):63-70. http://dx.doi.org/10.1590/S141382712003000100009.

21. Fernandes MH, Rocha VM. Impact of the psychosocial aspects of work on the quality of life of teachers. Rev Bras Psiquiatr. 2009;31(1):15-20. http://dx.doi.org/10.1590/S1516-44462009000100005. PMid:19506771.

22. Azevedo WF, Mathia LAST. Adição ao trabalho e qualidade de vida: um estudo com médicos. Einstein. 2017;15(2):130-5. http://dx.doi.org/10.1590/s1679-45082017ao3960. PMid:28767908.

23. Teixeira JRB, Boery EN, Casotti CA, Araújo TM, Pereira R, Ribeiro IJS, et al. Associação entre aspectos psicossociais do trabalho e qualidade de vida de mototaxistas. Cad Saude Publica. 2015;31(1):97-110. http://dx.doi.org/10.1590/0102-311X00214313. PMid:25715295.

24. Teles MA, Barbosa MR, Vargas AM, Gomes VE, Ferreira EF, Martins AM, et al. Psychosocial work conditions and quality of life among primary health care employees: a cross sectional study. Health Qual Life Outcomes. 2014;12(72):1-12. http://dx.doi.org/10.1186/1477-7525-12-72. PMid:24884707.

25. Nunes EA, Mascarenhas CHM. Qualidade de vida e fatores associados em trabalhadores do setor bancário. Rev Bras Med Trab. 2016;14(3):227-36. http://dx.doi.org/10.5327/Z1679-443520162216.

26. Araújo TM, Godinho TM, Reis EJFB, Almeida MMG. Diferenciais de gênero no trabalho docente e repercussões sobre a saúde. Cien Saude Colet. 2006;11(4):1117-29. http://dx.doi.org/10.1590/S141381232006000400032 .

27. Picoloto D, Silveira E. Prevalência de sintoma osteomusculares e fatores associados em trabalhadores de uma indústria metalúrgica de Canoas - RS. Cien Saude Colet. 2008;13(2):507-16. http://dx.doi.org/10.1590/ S1413-81232008000200026. PMid:18813567.

28. Kara D. Gender differences on perceptions of employee quality of working life in five star hotels in Turkey. World Appl Sci J. 2012;20(10):1383-8.

29. Almeida JR, Elias ET, Magalhães MA, Vieira AJD. Efeito da idade sobre a qualidade de vida e saúde dos catadores de material recicláveis de uma associação em Governador Valadares, Minas Gerais, Brasil. Cien Saude Colet. 2009;14(6):2169-79. http://dx.doi.org/10.1590/S1413-81232009000600024. PMid:20069185.

30. Steptoe A, Deaton A, Stone AA. Psychological wellbeing, health and ageing. Lancet. 2015;385(9968):640-8. http://dx.doi.org/10.1016/S0140-6736(13)61489-0. PMid:25468152.

31. Lee HS, Lee S, Park S, Baek Y, Youn JH, Cho DB, et al. The association between somatic and psychological discomfort and health-related quality of life according to the elderly and non-elderly. Qual Life Res. 2018;27(3):673-81. http://dx.doi.org/10.1007/s11136-017-1715-1. PMid:29027086.

32. Souza LK, Hutz CS. Relacionamentos pessoais e sociais: amizade em adultos. Psicol Estud. 2008;13(2):25765. http://dx.doi.org/10.1590/S1413-73722008000200008.

33. Souza DA, Cerqueira-Santos E. Redes Sociais e Relacionamentos de Amizade ao Longo do Ciclo Vital. Psicopedagogia. 2011;28(85):53-66.

34. Ribeiro AP, Souza ER, Atie $S$, Souza AC, Schilithz AO. A influência das quedas na qualidade de vida de idosos. Cien Saude Colet. 2008;13(4):1265-73. http://dx.doi.org/10.1590/S1413-81232008000400023. PMid:18813626.

35. Barrientos LA, Suazo SV. Fatores associados a qualidade de vida de enfermeiras hospitalares chilenas. Rev Lat Am Enfermagem. 2007;15(3):480-6. PMid:17653433.

36. Filippin NT, Martins JS, Libera LBD, Halberstadt BF, Severo AR. Qualidade de vida de sujeitos com doença de Parkinson e seus cuidadores. Fisioter Mov. 2014;27(1):57-66. http://dx.doi.org/10.1590/0103-5150.027.001. A006. 
37. Paula EA, Buschinelli JT, Maeno M, Costa RF. Qualidade de vida de trabalhadores com LER/DORT e lombalgia ocupacional atendidos no Cerest de Guarulhos, São Paulo. Rev Bras Saúde Ocup. 2016;41(19):1-11. http:// dx.doi.org/10.1590/2317-6369000120115.

38. Jahnke SA, Poston WSC, Haddock CK, Jitnarin N. Obesity and Incident Injury Among Career Firefighters in the Central United States. Obesity. 2013;21(8):1505-8. http://dx.doi.org/10.1002/oby.20436. PMid:23512940.

39. Levandoski G, Chiquito E, Oliveira AG, Zaremba CM. Qualidade de vida e composição corporal de soldados ingressantes no exército. Rev Bras Qual Vida. 2013;5(2):23-30. http://dx.doi.org/10.3895/S217508582013000200003.

40. Müller MR, Guimarães SS. Impacto dos transtornos do sono sobre o funcionamento diário e a qualidade de vida. Estud Psicol. 2007;24(4):519-28.

41. Pereira ÉF, Teixeira CS, Kothe F, Merino EAD, Daronco LSE. Percepção de qualidade do sono e da qualidade de vida de músicos de orquestra. Rev Psiquiatr Clin. 2010;37(2):48-51. http://dx.doi.org/10.1590/S010160832010000200003.

42. Cappuccio FP, Cooper D, D'Elia L, Strazzullo P, Miller MA. Sleep duration predicts cardiovascular outcomes: a systematic review and meta-analysis of prospective studies. Eur Heart J. 2011;32(12):1484-92. http:// dx.doi.org/10.1093/eurheartj/ehr007. PMid:21300732.

43. Crispim CA, Zalcman I, Dáttilo M, Padilha HG, Tufik S, Mello MT. Relação entre Sono e Obesidade: uma Revisão da Literatura. Arq Bras Endocrinol Metabol. 2007;51 (7):1041-9. http://dx.doi.org/10.1590/S000427302007000700004 . PMid:18157377.

44. Cauter EV, Spiegel K, Tasali E, Leproult R. Metabolic consequences of sleep and sleep loss. Sleep Med. 2015;9(Suppl 1):S23-8. 